\title{
BOTTOM-UP VERSUS TOP-DOWN HANDS-ON OPTIONS FOR MEASURING GHG AND POLLUTANTS IN SMART CITIES
}

\author{
SANDRINE QUÉRÉ \& ANNIE LEVASSEUR \\ Department of Construction Engineering, École de Technologie Supérieure, Canada
}

\begin{abstract}
Urbanized areas account for more than $70 \%$ of the carbon dioxide equivalent emissions. Their current greenhouse gas (GHG) emission is based on a bottom-up approach that adds the different sources of emissions (activity data multiplied by emission factors). Those current estimated inventories, based on statistics, are expensive and take months for data collection. Moreover, they raised scepticism for municipal decision-makers who are not certain how to understand and use them in urban policies planning support. Indeed, cities are lacking reliable, accessible information of a high standard on which to base GHG emission reduction decisions. To help smart cities measure and lower their emissions, another approach is currently under investigation: the top-down approach, based on real GHG measurements. In this paper, we present the current and potential hands-on options for measuring GHG: network of sensors, network of sensors coupled with atmospheric inversion modelling, and the laser beam system. We conclude by making recommendations for municipal decision makers to help them take ownership of in order to tackle climate change issues.
\end{abstract}

Keywords: city GHG emission inventory, sensors network, laser beam, air quality, smart city, empowering citizens.

\section{INTRODUCTION}

Cities account for more than $70 \%$ of energy-related global greenhouse gas (GHG) emissions [1]. Major cities in the world joined several initiatives such as the C40 Cities Climate Leadership Group and 100 Resilient Cities and took major commitments to lower their emissions. So far, cities GHG emission inventories are estimations obtained through a theoretical calculation. Those current inventories have incomplete and uncertain data. Moreover, they are of question of utility for mitigation decisions. Recently, a new trend appeared for smart cities to directly measure their GHG emissions to help them test and validate the impacts of their policy and urban decisions. In this paper, we discuss the different options tested by smart cities with their advantages and limitations.

In the past, cities have followed different protocols to establish their inventories, and recently, there is a tendency to use the Global Protocol for Community-Scale Greenhouse Gas Emission Inventories (GPC) [2] and the NAZCA platform [3]. The GPC inventory is an estimate of carbon dioxide $\left(\mathrm{CO}_{2}\right)$ emissions with all the other $\mathrm{GHG}$ converted into $\mathrm{CO}_{2}$ equivalent $\left(\mathrm{CO}_{2} \mathrm{e}\right)$. It is obtained through a theoretical calculation based on many assumptions that adds the different sources of emissions (activity data multiplied by emission factors).

\section{NEED OF MEASUREMENT DATA}

Traditional inventories have incomplete and uncertain data. For example, there is a lack of accurate statistics for the total amount of fuel used within cities [4]. And the uncertainty even builds up when extended to areas. For example, in 2010, estimates of GHG emissions in the Greater Toronto Area were available with the EDGAR [5] and FFDAS [6] inventories which provided a total of anthropogenic $\mathrm{CO}_{2}$ emissions of $1.42 \times 10^{8}$ and $6.04 \times 10^{7}$ tonnes, respectively. 
Those current inventories are expensive and take months for data collection. Moreover, they raise scepticism for municipal decision-makers who are not certain how to understand and use them in urban policies planning support. Indeed, cities are lacking reliable, accessible information of a high standard on which to base GHG emission reduction decisions. In brief, they need real GHG measurements to provide clarity, transparency and accountability which is called the top-down approach.

To help smart cities measure and lower their emissions, different options have been chosen. It is beyond the scope of this paper to talk about the use of satellites or aircraft measures as we focus only on hands-on options that could be used directly and easily by municipal leaders, once a pilot project would have been demonstrated conjointly with universities. The first option that we present is the implementation of sensors network. The second one combines sensors network with atmospheric inverse modelling. The third one uses the laser beam technology.

\section{NETWORK OF SENSORS}

Several cities have already implemented GHG and pollutant sensors. Here, we describe the projects implemented in the cities of Oakland, London, and Trondheim.

\subsection{Beacon Berkeley project}

The University of California at Berkeley installed a network of 50 low-cost sensors to measure GHG and pollutants $\left(\mathrm{CO}_{2}, \mathrm{CO}, \mathrm{NO}, \mathrm{NO}_{2}, \mathrm{O}_{3}, \mathrm{PM}\right)$. The sensors were spread over a $70 \mathrm{~km}^{2}$ grid at approximately $2 \mathrm{~km}$ from each other. This pilot project aimed at helping the city of Oakland to validate the effectiveness of carbon-reduction strategies. According to R. Cohen, Beacon Berkeley project leader, "Real time observations will enable rapid verification of the effectiveness of policy and compliance with treaties and other agreements and commitments". The major outcome of that project remained educational outreach on climate science as most of the sensors were displayed atop of schools [7].

\subsection{London network}

The city of London took a big step in 2018 to improve its air quality thanks to his mayor Sadiq Aman Khan. 100 fixed sensors have been installed on lampposts and buildings in the worst polluted areas and two Google street cars are driving across the city. The fixed sensors measure GHGs and pollutants $\left(\mathrm{CO}, \mathrm{O}_{3}, \mathrm{NO}_{2}, \mathrm{PM}\right)$.

The Google street cars driving on the streets of London are equipped with sensors that already mapped the city of San Francisco with a $30 \mathrm{~m}$ resolution. The sensors were provided by the company Aclima which measured $\mathrm{NO}_{2}, \mathrm{NO}$ and black carbon. In September 2018, Google and Aclima announced planning the measurement of $\mathrm{CO}$ and $\mathrm{CO}_{2}, \mathrm{O}_{3}$ and particles PM. The data are available to the public via Google BigQuery. According to Google, the data should help city decision makers to take the best mitigation measures. Aclima received a new funding of \$24 million for this project.

In addition to that fixed and mobile network orchestrated by the city of London, mayor Khan's objective was to raise citizen awareness. To that end, the city of London published a guide available to anyone wanting to monitor air quality. This guide describes the different sensors that are available to be bought on the market, how to collect the data, understand them and send them back to the city [8]. Mayor Khan believes that empowering Londoners with devices that provide them with a capacity of directly measuring what they are breathing is very important. 
An example of the pollutant sensors that could be chosen by the Londoners are the PurpleAir sensors because the system is already connected to internet [9]. The PurpleAir sensors, developed in North America, are already used by the younger citizens, tech-savvy residents and parents concerned with air quality around schools. Those sensors give the real time and week average data for particles $\mathrm{PM}_{1.0}(<1 \mu \mathrm{m}), \mathrm{PM}_{2.5}(<2.5 \mu \mathrm{m})$ and $\mathrm{PM}_{10}(<10 \mu \mathrm{m})$. As an example, as shown in Fig. 1, are the $\mathrm{PM}_{2.5}$ data for the Montreal area on 9th September 2020. The data for Fabreville Laval provides an index of 4 which indicates that air quality is considered satisfactory, and air pollution poses little or no risk. The data for downtown Montreal provides indexes of 63 and 64, which show that air quality is acceptable; however, if they are exposed for 24 hours, there may be a moderate health concern for people who are unusually sensitive to air pollution.

\subsection{Trondheim network}

Scandinavian cities, Trondheim in Norway and Vejle in Denmark, have chosen to test GHG and pollutant sensors within the Carbon Track and Trace (CTT) project [10]. CTT couples low-cost, open-source sensors to an Internet of Thing (IoT) platform. The main goal was to provide cities with a real-time GHG measurement capacity in order to directly measure the impacts of their policy and urban decisions. The second objective was to implement a semiautonomous system for city GHG emission inventories.

That project started through the launch of a 24-hour climathon in 2016. After two years of project, the main outcome for the city of Trondheim was the possibility of discussing urban planning issues and see, for example, how pollution level would affect their decision making. They were also able to choose the location of air quality sensors according to new data sets such as road networks and the density of buildings.

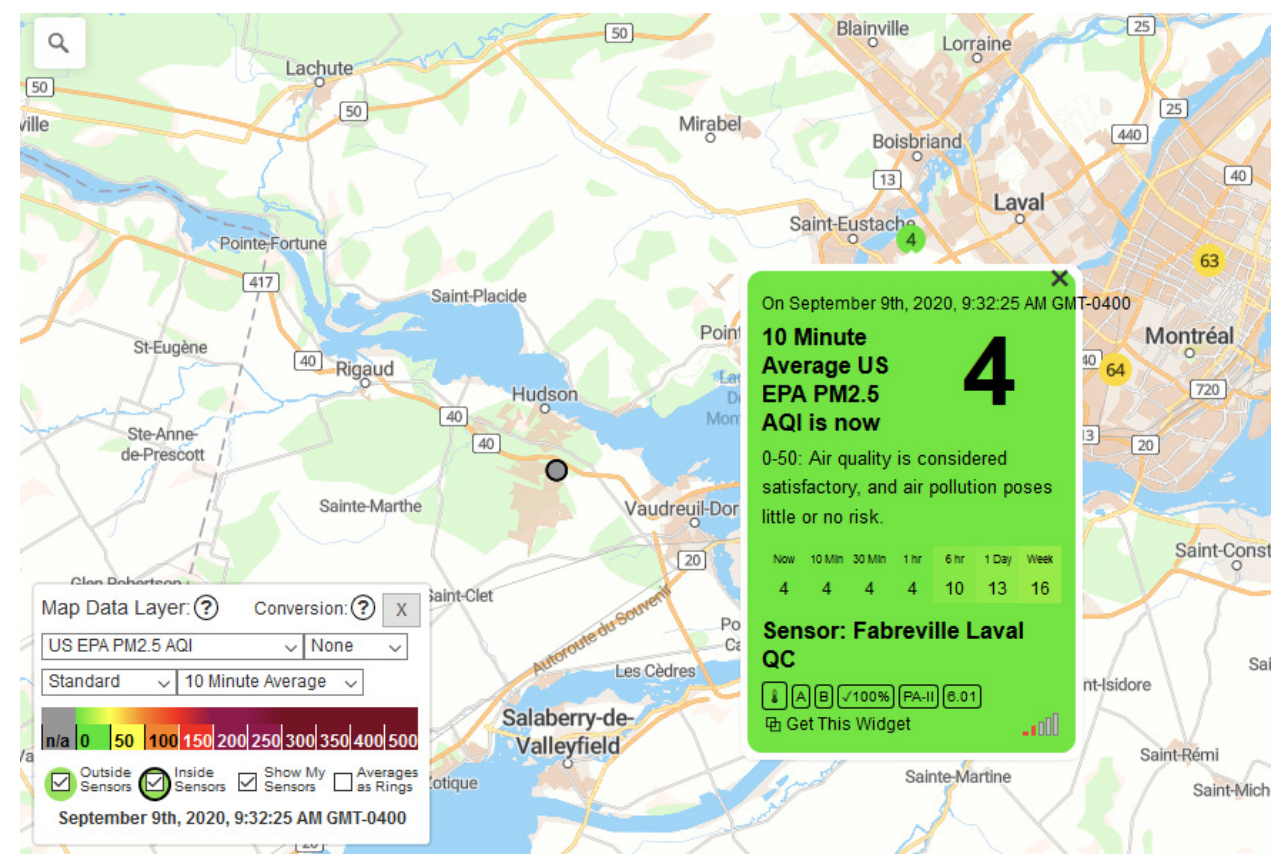

Figure 1: Air quality measurements for particles $\mathrm{PM}_{2.5}$ with PurpleAir Sensors. 
The main conclusion of their 2018 paper was that "Integration into decision support systems is a far goal". They understood that trying to link a $\mathrm{CO}_{2}$ concentration curve with a traffic jam factor is much more complicated than a simple correlation as other elements account. $\mathrm{CO}_{2}$ dynamics has to be properly modelled to identify its anthropogenic sources of emissions (ground transportation, residence, industry). They concluded their paper by stating that they would in their future work also include wind speed, temperature, weather conditions and seasonal patterns.

\subsection{Main limitation of the sensors network}

In brief, the main disadvantage of this method is the limited representativeness of total urban emissions and the impossibility of identifying the sources of emissions. Each site is influenced by both local and remote sources and no modelling is performed to identify the different sources.

\section{SENSORS NETWORK COMBINED WITH ATMOSPHERIC INVERSE MODELLING}

The second option smart cities have chosen to measure and lower their GHG emissions is to combine GHG sensor networks with atmospheric inverse modelling coupled with meteorological and chemical transport models. The main objectives of this methodology called CarboCount City were to estimate city GHG emission inventories independently from the traditional inventories, to decrease the current inventory uncertainties and also to identify the sources of emissions by using inverse atmospheric modelling. This method has already been implemented in test-bed cities, for example in Paris [11], [12] and Indianapolis [13], [14].

\subsection{Atmospheric inverse modelling methodology}

Emissions from the current GHG inventory are inputs for the combined meteorological and chemical transport model. The outputs provide estimates of $\mathrm{CO}_{2}$ concentrations that are compared to the observations given by the sensors. The difference (estimates-observations) is used by an inversion technique that uses probabilistic tools to give the emissions of an updated inventory that would reduce this difference.

\subsection{INFLUX project}

In Indianapolis, the INFLUX project (Indianapolis Flux Experiment) main objective was to provide additional constraints for the compilation of inventories as well as elements of control (verification) of budgets obtained with conventional inventories. The INFLUX project showed the ability to estimate urban GHG emission inventory from three sources: a previous GHG inventory, airborne and tower-based measurements coupled with atmospheric inversion modelling.

Nevertheless, the results show some differences across methods that still have to be resolved. As the INFLUX project includes aircraft data and that research is still underway to resolve discrepancies, the model is not a hands-on system for cities [14]. 


\subsection{Paris network}

In 2015, the city of Paris received a lot of spotlight by hosting the COP21, the 21st Conference of the Parties of the United Nations Framework Convention on Climate Change (UNFCCC).

For the CarboCount City project implemented in Paris, only three expansive stations surrounding the city were used in the inverse modelling. They were located around the city, on buildings between 4-9 $\mathrm{m}$ above ground level [12].

The study used gradients between upwind and downwind concentrations because they are the best source of urban concentration data for inversion purposes. The most robust data for this study used only afternoon concentration gradients to avoid biases in the vertical mixing during nighttime, morning and evenings when chemical transport models cannot represent correctly the planetary boundary layer which is the lowest layer of the atmosphere that collects $\mathrm{CO}_{2}$ emissions from cities [15]. A second condition was to sort the $\mathrm{CO}_{2}$ gradients between pairs of stations along the wind direction. A third condition imposed a threshold on wind speeds (larger than $3 \mathrm{~m} / \mathrm{s}$ ) as chemical transport models have difficulties on modelling the lower speeds.

All in all, those conditions explained why $92 \%$ of the total hourly observations were removed. The main outcome of this paper was that the inverted $\mathrm{CO}_{2}$ concentration emissions followed the monthly mean heating curve for the center of Paris [11].

\subsection{Other studies based on the Paris network}

Another study based on the Paris network and using observing system simulation experiments (OSSES) demonstrated the value of dense network in decreasing the uncertainty of the current inventories and in quantifying emissions for different sources of activity [16]. For example, the conventional AIRPARIF 2008 inventory based on a $1 \mathrm{~km}$ resolution is assigned a $20 \% 1 \sigma$ uncertainty in the monthly estimate of the total emissions from île de France [17]. By simulating a network of 10 stations, the OSSES experiment estimated a monthly $1 \sigma$ uncertainty around $11 \%$, which means a $42 \%$ uncertainty reduction. By simulating higher number of sensors, they demonstrated that the total and sectorial uncertainties were decreasing when the number of sensors was increasing.

Other studies have used observations of carbon isotopes and co-emitted pollutants to better quantify the sources of emissions [18], [19].

\subsection{Main limitation of the atmospheric inverse modelling}

Atmospheric inversion modelling aims at determining GHG flux in and out of the atmosphere. The sensor networks are ideally located around the city to compile urban inventories and not inside. According to reference [11], the inversion method still needs improvements to reach a satisfactory agreement between the measured concentrations and estimations. Even if studies on paper show that uncertainties decrease with the number of expansive stations, from a practical point of view, a network of low-cost sensors at a resolution of $100 \mathrm{~m}$ would need to be implemented to try to get an idea of the anthropogenic emissions. This second network would also increase dramatically the cost of those experiment. Designing an observing network suitable for urban policies needs to gain in maturity before being implemented [20]. 


\section{LASER BEAM TECHNOLOGY}

In order to overcome the gaps from the inverse atmospheric method, a new technology was tested in Paris for COP21. That technology, called Greenhouse gas Laser Imaging Tomography Experiment (GreenLITE), is based on a laser absorption spectroscopy system. Its main advantage is to measure 2-D spatial $\mathrm{CO}_{2}$ concentrations [21].

\subsection{Pilot project in Paris}

This ground-based system was deployed over Paris for one year between November 2015 and November 2016 and utilized two laser transceivers and fifteen receivers covering $\sim 25 \mathrm{~km}^{2}$. The system provided the city of Paris with a yearlong of 24/7 real-time measurements capability. GreenLITE demonstrated its capacity of mapping $\mathrm{CO}_{2}$ urban concentrations with a resolution of $\sim 200 \mathrm{~m}$. The near real-time measurement of $\mathrm{CO}_{2}$ concentrations provides a first-order estimate of local changes in $\mathrm{CO}_{2}$ flux, but cannot provide absolute flux values.

The measurements as well as atmospheric data (temperature, pressure, humidity, wind speed and direction) were displayed on a web interface. Initial assessments of the measurement against in situ instrumentation and modeled values showed very good agreement.

GreenLITE succeeded in mapping diurnal cycles linked to anthropogenic activity. For example, the laser beam technology was able to show the alternate traffic circulation policy implemented by the city of Paris to reduce $\mathrm{CO}_{2}$ emissions during pollution peak days, as well as differences in weekday versus weekend diurnal cycles.

This technology provides spatially averaged measurements which may benefit atmospheric inversion modelling as the uncertainties obtained with GreenLITE are lower than those obtained with sensors [22] (personal communication with Jeremy Dobler).

GreenLITE demonstrated a similar system capability for methane. This methodology seems therefore to be promising in helping cities to evaluate their mitigation measures.

\subsection{Main limitation of the laser beam technology}

This technology measures the total $\mathrm{CO}_{2}$ concentration along path observations between transceivers and receivers and cannot identify the different anthropogenic sources (ground transportation, industry, residence). Nevertheless, the $\mathrm{CO}_{2}$ concentration map measures local carbon footprints and allows to know where the sources are located.

\section{CHOOSING THE BEST OPTION}

In order to choose the best system adapted to the city, stakeholders have firstly to identify their needs and objectives, as well as the mitigation measures that could be validated thanks to hands-on measurement GHG system.

As the perfect option able to identify different anthropogenic sources does not seem to exist yet, the development of innovative techniques to use new data sources is necessary. Those techniques might help to reconciliate the bottom-up and top-down approaches.

The implementation of a support process for municipal decision makers will help to take appropriate urban mitigation decisions, which could be, for example, validating the restriction of traffic on certain roads and see where the circulation adjustments happen, measuring GHG emissions for specific events, monitoring pollution at road interchanges or near industrial centers. 
By enrolling their citizens as London did, cities empower them in being informed and taking action. Citizens can use their new knowledge in pollution to improve the air quality for their neighborhood, around the schools, around their workplaces and for their displacements.

\section{CONCLUSIONS}

Choosing the best measurement system for a city means identifying the needs and objectives, as well as evaluating the project feasibility and limitations. Some cities like London and Trondheim have chosen to record some trends and are now faced by the limited information given by the collected data. What do they mean and how to use them?

Other cities like Indianapolis or Paris have implemented GHG sensors coupled with a 3D sophisticated atmospheric inversion model. This method is able to provide additional constraints for the compilation of inventories. Stations placed at strategic locations determine the urban concentration data in the direction of the wind. A second network of low-cost sensors has to be implemented to measure the high-resolution urban flux and the anthropogenic emissions. No city has yet tried to implement a coherent and complete set of stations and low-cost sensors. The ultimate objective of estimating city GHG inventories independently from the traditional inventories remains a far goal. This inversion method is an open area of research and the path is still long before a high-density sensor network could test and validate municipal emission reduction measures.

A third option that we discussed is the laser beam technology that provides cities with a capacity to map $\mathrm{CO}_{2}$ concentrations in real-time with a resolution of $\sim 200 \mathrm{~m}$. This system has already demonstrated its ability in validating urban mitigation measures and seems to be promising in regard to their effectiveness of policy implementation in both health and environmental scopes.

Our main recommendations concern the reconciliation of the bottom-up and top-down approaches, the development of innovative techniques to use new data sources and the implementation of a support process for decision makers so that they can take appropriate urban mitigation decisions and reach their GHG emission reduction targets.

\section{ACKNOWLEDGEMENTS}

S.Q. acknowledges support from the Centre interdisciplinaire en opérationnalisation du développement durable (CIRODD), from la Maison de l'innovation sociale (MIS) and from Jalon Montréal, l'Institut des transports intelligents.

\section{REFERENCES}

[1] International Energy Agency, World Energy Outlook, 2008. www.iea.org/media/ weowebsite/2008-1994/WEO2008.pdf. Accessed on: 15 Aug. 2020.

[2] Fong, W. et al., Global Protocol for Community-Scale Greenhouse Gas Emission Inventories - An Accounting and Reporting Standard for Cities. World Resources Institute, C40 Cities Climate Leadership Group and Local Governments for Sustainability (ICLEI), 2014. http://ghgprotocol.org/files/ghgp/GHGP_GPC.pdf. Accessed on: 15 Aug. 2020.

[3] Partnership for Global Climate Action. https://climateaction.unfccc.int/. Accessed on: 19 Aug. 2020.

[4] $\mathrm{Wu}, \mathrm{L}$. et al., What would dense atmospheric observation networks bring to the quantification of city $\mathrm{CO}_{2}$ emissions? Atmospheric Chemistry and Physics, 16(12), pp. 7743-7771, 2016. 
[5] Emission Database for Global Atmospheric Research Release, Version 4.2 of the European Commission. Joint Research Centre (JRC)/Netherlands Environmental Assessment Agency (PBL), 2010. http://edgar.jrc.ec.europa.eu. Accessed on: $10 \mathrm{Jul}$. 2020.

[6] Fossil Fuel Data Assimilation System (FFDAS), 2010. http://hpcg.purdue.edu/ FFDAS/map.php. Accessed on: 5 Jul. 2020.

[7] Beacon Berkeley Project, 2012. http://beacon.berkeley.edu/about/. Accessed on: 5 Jun. 2020.

[8] City of London, Guide for monitoring air quality in London, 2018. www.london.gov.uk/sites/default/files/air_quality_monitoring_guidance_january_20 18.pdf. Accessed on: 15 Aug. 2020.

[9] PurpleAir sensors. www2.purpleair.com/collections/air-quality-sensors. Accessed on: 9 Sep. 2020.

[10] Ahlers, D. et al., Analysis and visualization of urban urban emission measurements in smart cities. Proceedings of the 21st International Conference on Extending Database Technology (EDBT), 2018.

[11] Bréon, F.M. et al., An attempt at estimating Paris area $\mathrm{CO}_{2}$ emissions from atmospheric concentration measurements. Atmospheric Chemistry and Physics, 15, pp. 1707-1724, 2015.

[12] Staufer, J. et al., The first 1-year-long estimate of the Paris region fossil fuel $\mathrm{CO}_{2}$ emissions based on atmospheric inversion. Atmospheric Chemistry and Physics, 16(22), pp. 14703-14726, 2016.

[13] Lauvaux, T. et al., High-resolution atmospheric inversion of urban $\mathrm{CO}_{2}$ emissions during the dormant season of the Indianapolis Flux Experiment (INFLUX). Journal of Geophysical Research - Atmospheres, 121(10), pp. 5213-5236, 2016.

[14] Davis, K.J. et al., The Indianapolis Flux Experiment (INFLUX): A test-bed for developing urban greenhouse gas emission measurements. Elementa-Science of the Anthropocene, 5, p. 21, 2017.

[15] Steeneveld, G.J., Vilà-Guerau de Arellano, J., Holtslag, A.A.M., Mauritsen, T., Svensson, G. \& de Bruijn, E.I.F., Evaluation of limited-area models for the representation of the diurnal cycle and contrasting nights. In CASES-99, Journal of Applied Meteorology and Climatology, 47, pp. 869-887, 2008.

[16] $\mathrm{Wu}, \mathrm{L}$. et al., What would dense atmospheric observation networks bring to the quantification of city $\mathrm{CO}_{2}$ emissions? Atmospheric Chemistry and Physics, 16(12), pp. 7743-7771, 2016.

[17] AIRPARIF: Inventaire des émissions en Île-de-France. Résultats - année 2008, Tech. rep., AIRPARIF, Surveillance de la Qualité de l'Air en Île-de-France, 2012. www.airparif.asso.fr/_pdf/publications/Rinventaire-2008-120217.pdf. Accessed on: 15 Aug. 2020.

[18] Ammoura, L. et al., Atmospheric measurements of ratios between $\mathrm{CO}_{2}$ and co-emitted species from traffic: A tunnel study in the Paris megacity. Atmospheric Chemistry and Physics, 14, pp. 12871-12882, 2014.

[19] Pugliese, S.C. et al., High-resolution quantification of atmospheric $\mathrm{CO}_{2}$ mixing ratios in the Greater Toronto Area, Canada. Atmospheric Chemistry and Physics, 18, pp. 3387-3401, 2018.

[20] Bureau International des Poids et Mesures, Workshop on Global to Urban Scale Carbon Measurement, 2015. www.bipm.org/utils/common/pdf/carbon-workshopreport-20151120.pdf. Accessed on: 10 Aug. 2020. 
[21] Dobler, J., Zaccheo, T. S., Pernini, T., Blume, N. \& Barun, M., Greenlite ${ }^{\mathrm{TM}}$ : A year of carbon dioxide monitoring over Paris, France, and recent progress in monitoring methane. Proceedings of the 28th International Laser Radar Conference, 176, p. 05013, 2018.

[22] Dobler, J., Personal communication, 18 December 2018, lead on the GreenLITETM system, Scientist Systems Engineering, Space And Intelligence Systems, Harris Corporation: Fort Wayne, USA. 